\title{
EDITORIAL OPEN Novel targeted drugs approved by the NMPA and FDA in 2019
}

Signal Transduction and Targeted Therapy (2020)5:65

https://doi.org/10.1038/s41392-020-0164-4

In 2019, the FDA's Center for Drug Evaluation and Research (CDER) approved 48 novel drugs [https://www.fda.gov/drugs/new-drugsfda-cders-new-molecular-entities-and-new-therapeutic-biologicalproducts/novel-drug-approvals-2019], ${ }^{1}$ which was fewer than the all-time record of 62 NTD approvals in $2018,{ }^{2}$ but it was still a fruitful year. Among these approved drugs, 39 were targeted drugs (Table 1), including 27 small molecules, 3 antibody-drug conjugates (ADCs), 1 RNA interference (RNAi) therapy, 1 antisense oligonucleotide, 4 monoclonal antibodies (mAbs), 1 recombinant fusion protein, and 2 synthetic peptide analogs. The targets included kinases, ion channels, exons, enzymes, and receptors. Oncology, which remains the most important drug discovery area, accounted for $23 \%$ (9/39) of the targeted drug approvals.

Small molecule drugs play an important role in fighting diseases. Although the development of small molecules has slowed slightly in recent years, the 27 small molecule targeted drugs approved in 2019 accounted for nearly $70 \%$ of the total number of approved targeted drugs. Small molecule drugs have the advantages of oral bioavailability, pharmacokinetics, drug delivery, production cost, etc., which facilitate the development of this class of drugs, and this comparative advantage will continue in the near future. In addition, small molecules may be used in conjunction with new types of therapies, such as antibody-drug conjugates (ADCs).

In 2019, there was an increase in the number of approved ADCs: polatuzumab vedotin-piiq (Polivy) for relapsed or refractory diffuse large B-cell lymphoma, enfortumab vedotin-ejfv (Padcev) for refractory bladder cancer and fam-trastuzumab deruxtecannxki (Enhertu) for metastatic breast cancer. ADCs comprise a monoclonal antibody and cytotoxic agents conjugated via a chemical linker. The specificity of mAbs allows the chemotherapy agents to be selectively delivered to targeted cancer cells, thereby reducing toxicity. Importantly, mAbs such as trastuzumab not only show specificity but also have anticancer effects. To date, seven ADCs have been approved by the FDA for clinical use, and over 100 ADCs are in clinical development. ${ }^{3}$

Two synthetic peptide analogs were approved this year, bremelanotide and afamelanotide. Peptide-based therapy has been applied in various diseases, such as infectious diseases, allergic diseases, autoimmune diseases, sexual dysfunction, and fibrosis. Many efforts have been made to discover novel bioactive peptides. There is much potential for peptide-based therapy.

Another surprising newly emerging field in 2019 was gene therapy. In 2019, two gene therapy products were approvedGivlaari (givosiran) from Alnylam Pharmaceuticals and Vyondys 53 (golodirsen) from Sarepta Therapeutics. Givlaari is an RNA interference (RNAi) therapeutic that targets aminolevulinic acid synthase 1 (ALAS1) to treat acute hepatic porphyria (AHP). This is the second RNAi therapy approved by the FDA; Onpattro (patisiran) was the first. Onpattro was also developed by Alnylam
Pharmaceuticals and was approved by the FDA in 2018 to treat hereditary TTR-mediated amyloidosis. Both drugs use enhanced stabilization chemistry (ESC)-GalNAc conjugate technology. Vyondys 53 is an antisense oligonucleotide developed from Sarepta's phosphorodiamidate morpholino oligomer (PMO) platform; it was approved to treat Duchenne muscular dystrophy (DMD) patients who have a confirmed mutation of the dystrophin gene that causes exon 53 skipping. In the future, more gene therapies currently under development are likely to be approved in the upcoming years, which would bring hope to individuals with severe, life-threatening diseases or rare diseases.

The FDA approved Brukinsa (zanubrutinib) capsules from BeiGene USA, Inc., for the treatment of adult patients with mantle cell lymphoma who received at least one prior therapy. Brukinsa is the first novel anticancer drug developed by a Chinese company and approved by the FDA. It was granted Accelerated Approval, Breakthrough Therapy designation, and Orphan Drug designation. Unfortunately, BeiGene, Ltd., announced that the new drug application (NDA) for zanubrutinib for the treatment of patients with relapsed/refractory mantle cell lymphoma (MCL) was accepted by the National Medical Products Administration (NMPA) on $08 / 27 / 2018$, but zanubrutinib has not yet been approved.

On 08/30/2018, the China Food and Drug Administration (CFDA) changed its name to the National Medical Products Administration (NMPA), which is administered by the State Administration for Market Regulation (SAMR). In 2019, the NMPA approved 51 new drugs. Herein, we only summarize the eight innovative targeted drugs developed by the Chinese pharmaceutical industry (Table 2), including five small molecules, one antibiotic, one synthetic peptide analog, and two mAbs.

Two cancer immunotherapy drugs that target PD-1 have been approved, camrelizumab from Jiangsu Hengrui Medicine Co. and tislelizumab from BeiGene. Camrelizumab and tislelizumab are humanized lgG4 anti-PD-1 monoclonal antibodies that block the binding of PD-1 to its ligands. The first PD-1 inhibitor to hit the market was pembrolizumab (Keytruda), which was approved by the FDA in 2014. Since, ten PD-1/PD-L1 cancer immunotherapy drugs have come on the market worldwide, four of which were developed by Chinese pharmaceutical companies; these drugs are camrelizumab, tislelizumab, and sintilimab developed by Innovent Biologics and Eli Lilly and toripalimab developed by Shanghai Junshi Bioscience Co., Ltd. Following the FDA approval of Brukinsa (zanubrutinib), tislelizumab was the first drug developed by BeiGene to be approved in China. These young Chinese pharmaceutical companies show great potential in drug discovery, especially for novel targets. As China is the world's second-largest pharmaceutical market, we expect to see an increase in the number of novel drugs developed in China.

New classes of drugs developed using new technologies provide new treatment options and hope to patients with fatal diseases. For example, the cancer mortality rate in the US declined by $29 \%$ from 1991 to 2017 . This success is partially due to targeted therapies such as the BRAF inhibitor Zelboraf (vemurafenib) and the anti-CTLA4 antibody Yervoy (ipilimumab). ${ }^{4}$ In addition, emerging innovative therapeutic approaches such as CAR-T cell 


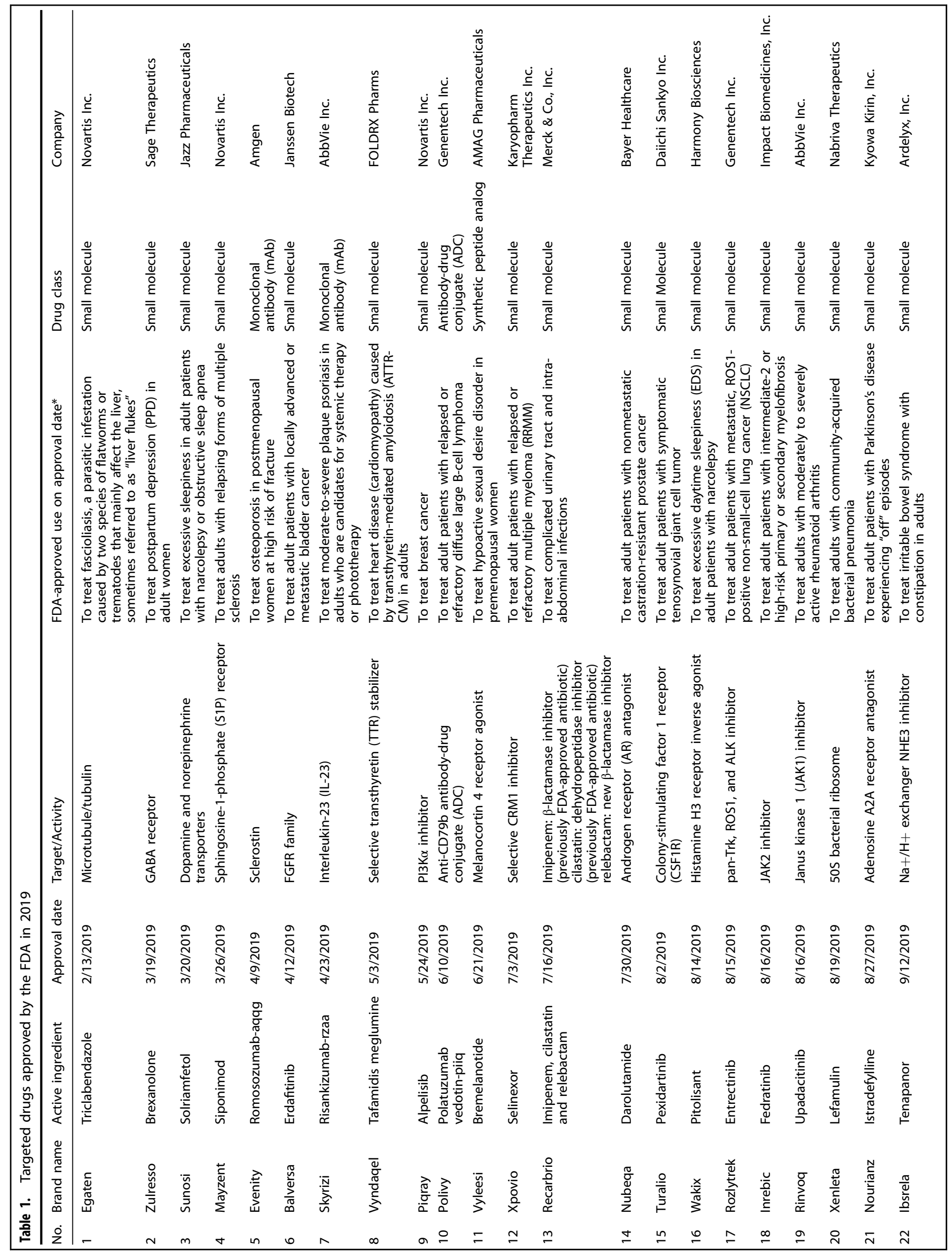




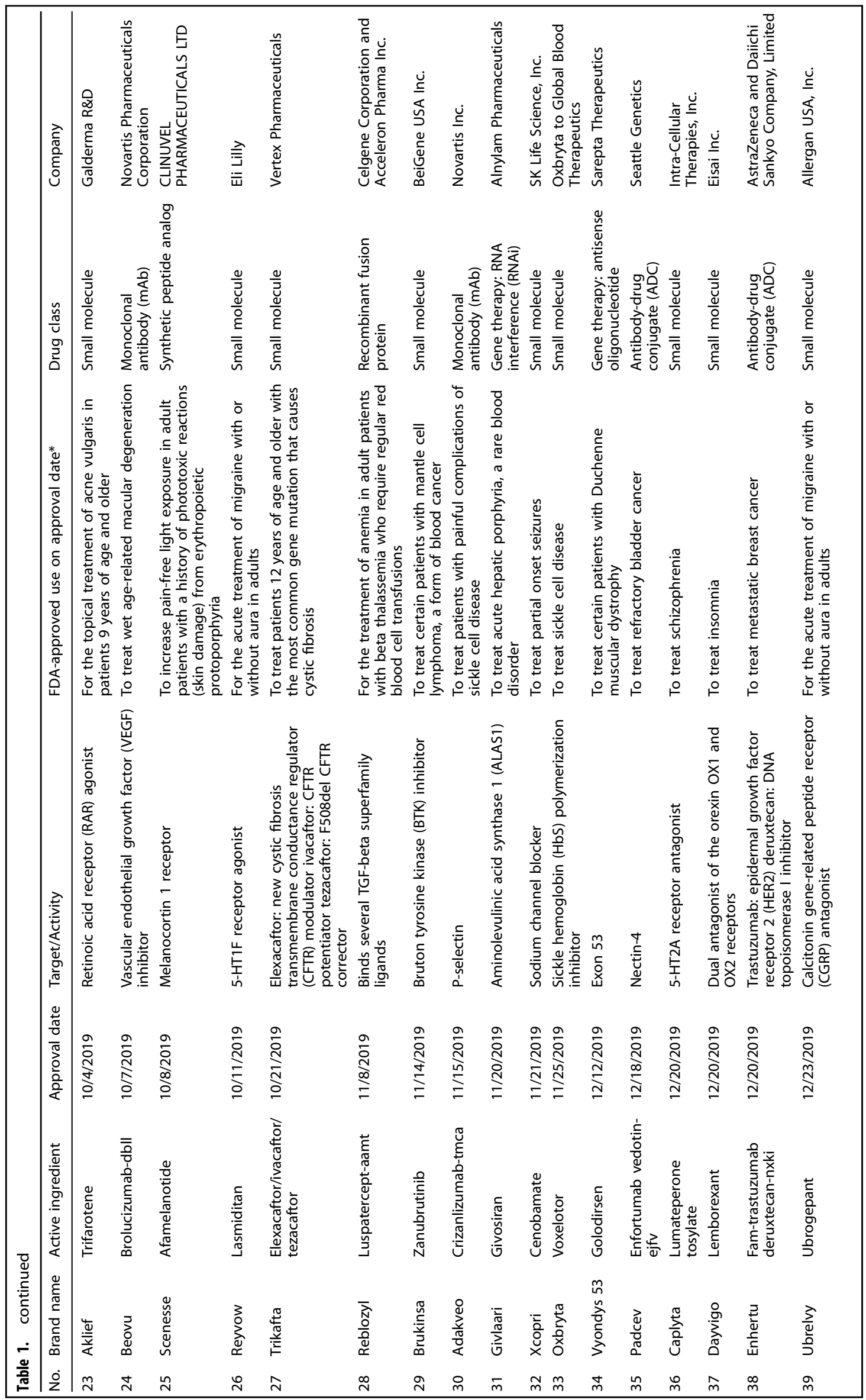


therapy for cancer and CRISPR-Cas9 gene editing could potentially make a difference for patients.

In 2020, Signal Transduction and Targeted Therapy aims to be the leading forum for research addressing unmet medical needs, including cancer, immune disorders, infectious diseases, virus such as SARS-CoV-2, diabetes, cardiovascular diseases, inflammation, central nervous system diseases, and other pathologies. We expect to publish papers on the discovery and development of new targets and therapeutic options that may have a significant impact on healthcare in the future.

\section{ADDITIONAL INFORMATION}

Competing interests: The authors declare no competing interests.

Wenjing Wang ${ }^{1}$ and Qiu Sun ${ }^{1}$

${ }^{1}$ State Key Laboratory of Biotherapy, Cancer Center, West China Hospital, Sichuan University and Collaborative Innovation Center for Biotherapy, 610041 Chengdu, China Correspondence: Wenjing Wang (wangwenjing@wchscu.cn) or Qiu Sun (sunqiu@wchscu.cn)

\section{REFERENCES}

1. Mullard, A. 2019 FDA drug approvals. Nat. Rev. Drug Discov. 19, 79-84 (2020).

2. Mullard, A. 2018 FDA drug approvals. Nat. Rev. Drug Discov. 18, 85-89 (2019).

3. Chau, C. H., Steeg, P. S. \& Figg, W. D. Antibody-drug conjugates for cancer. Lancet 394, 793-804 (2019).

4. Siegel, R. L., Miller, K. D. \& Jemal, A. Cancer statistics, 2020. Cancer J. Clin. 70, 7-30 (2020).

(C) Open Access This article is licensed under a Creative Commons Attribution 4.0 International License, which permits use, sharing, adaptation, distribution and reproduction in any medium or format, as long as you give appropriate credit to the original author(s) and the source, provide a link to the Creative Commons license, and indicate if changes were made. The images or other third party material in this article are included in the article's Creative Commons license, unless indicated otherwise in a credit line to the material. If material is not included in the article's Creative Commons license and your intended use is not permitted by statutory regulation or exceeds the permitted use, you will need to obtain permission directly from the copyright holder. To view a copy of this license, visit http://creativecommons. org/licenses/by/4.0/.

(c) The Author(s) 2020 